\title{
Yersinia mollaretii
}

National Cancer Institute

\section{Source}

National Cancer Institute. Yersinia mollaretii. NCI Thesaurus. Code C86859.

A species of facultatively anaerobic, Gram negative, rod shaped bacteria in the phylum Proteobacteria. This species is positive for urease, ornithine decarboxylase, and pyrazinamidase, motile and negative for indole, citrate, and oxidase. It is able to ferment sucrose, sorbose, cellobiose, and xylose but not rhamnose, melibiose, or fucose. Y. mollaretii is found in many environments and is not known to be a pathogen. 